\title{
Cardio-protective and Anti-atherosclerosis Effect of Crocetin on Vitamin D3 and HFD-induced Atherosclerosis in Rats
}

\author{
Laizeng $\mathrm{Yu}^{1 *}$, Ruihua $\mathrm{Gao}^{2}$, Xuewen Song ${ }^{1}$, Xuechao $\mathrm{Li}^{3}$, and Jiacheng $\mathrm{Zhu}^{1}$ \\ ${ }^{1}$ Internal Medicine of Traditional Chinese Medicine, Shandong Xinzhonglu Hospital of Traditional Chinese Medicine, Jinan, 250013, CHINA \\ ${ }^{2}$ Nursing College, Nanchang Medical College, Wanli District, Nanchang City, Jiangxi Province, 330014, CHINA \\ ${ }^{3}$ Department of Traditional Chinese Medicine, Fenghuangling Street Hospital, Fenghuangling Street Office, Linyi City, Shandong Province, \\ 276000, CHINA
}

\begin{abstract}
Cardiovascular disease (CVD) is a chronic disease and causes the highest rate of death globally. CVD-related deaths account for $\mathbf{8 0 \%}$ of all deaths in low and middle-income countries, such as China. Crocetin (CT), a carotenoid phytoconstituent already confirm their anti-inflammatory and antioxidant effects in various diseases animal models. In the study, we make effort to access the cardio-protective effect of Crocetin against vitamin D3 and high fat induced atherosclerosis in rats and scrutinize the underlying mechanism. Sprague Dawley (SD) rats were used in this study and rats were divided into different groups and high fat diet and vitamin $D$ was used for induction the atherosclerosis. The rats were received oral administration of crocetin $(5,10$ and $15 \mathrm{mg} / \mathrm{kg})$ and simvastatin $(0.5 \mathrm{mg} / \mathrm{kg})$ until 30 days. At the end of the experimental period, lipid, cardiac markers, anti-inflammatory, antioxidant, pro-inflammatory cytokines and atherogenic index were estimated. The mRNA expression of Intercellular adhesion molecule-1 (ICAM1), Monocyte Chemoattractant Protein-1 (MCP-1) and vascular cell adhesion molecule 1 (VCAM-1) in aortic tissue of the atherosclerotic rats. Crocetin significantly reduced the aortic membrane thickness and platelet aggregation rates. Crocetin also dose-dependently reduced total cholesterol (TC), very low-density lipoprotein (VLDL), triacylglycerol (TG), low-density lipoprotein (LDL) and augmented the level of highdensity lipoprotein (HDL) level. Additionally, Crocetin significantly $(p<0.001)$ abridged the level of malonaldehyde (MDA) and augmented the level of superoxide dismutase (SOD), catalase (CAT), reduced glutathione (GSH) and glutathione peroxidase (GPx). Furthermore, Crocetin significantly $(p<0.001)$ dosedependently reduced the levels of pro-inflammatory cytokines and inflammatory mediators. Crocetin attenuated mRNA expression of VCAM-1, ICAM-1 and MCP-1. Crocetin had anti-atherosclerosis and cardio-protective effects on vitamin D3 and high fat induced atherosclerosis in rats through antiinflammatory and antioxidant mechanisms.
\end{abstract}

Key words: cardio-protective, anti-atherosclerosis, crocetin, Vitamin D3, high fat diet

Abbreviations: CVD; Cardiovascular disease, CT; Crocetin, SD; Sprague Dawley, ICAM-1; Intercellular adhesion molecule-1, MCP1; Monocyte Chemoattractant Protein-1, VCAM-1; Vascular cell adhesion molecule 1, TC; Total Cholesterol, VLDL; Very lowdensity lipoprotein, TG; Triacylglycerol, LDL; Low-density lipoprotein, HDL; High-density lipoprotein, SOD; Superoxide dismutase, CAT; Catalase, GSH; Reduced glutathione, GPx; Glutathione peroxidase, as; Atherosclerosis, EC; Endothelial cells, SMC; Smooth muscle cells, NO; Nitric oxide, Enos; Enzyme nitric oxide synthase, MDA; Malondialdehyde, LDH; Lactate dehydrogenase, NO; Nitric oxide, hs-CRP; C-reactive protein, ET-1; Endothelin 1, TXB2; Thromboxane B2, cTnI; Cardiac troponin I, AST; Aspartate aminotransferase, ALT; Alanine aminotransferase, ALP; Alkaline phosphatase, IL-1 $\beta$; Interleukin-1 $\beta$, IL-6; Interleukin-6, TNF- $\alpha$; Tumor necrosis factor- $\alpha$, LCAT; Lecithin cholesterol-O-acyltransferase \footnotetext{
Chinese Medicine, Jinan, 250013, CHINA

E-mail: ylz13210539373@ sina.com ORCID ID: https://orcid.org/0000-0002-6501-3625

Accepted June 22, 2021 (received for review May 21, 2021)

Journal of Oleo Science ISSN 1345-8957 print / ISSN 1347-3352 online

http://www.jstage.jst.go.jp/browse/jos/ http://mc.manusriptcentral.com/jjocs
}

*Correspondence to: Laizeng Yu, Internal Medicine of Traditional Chinese Medicine, Shandong Xinzhonglu Hospital of Traditional

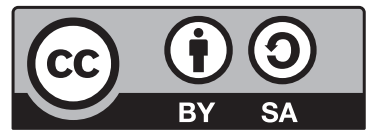




\section{Yu, Q. Kong, R. Gao et al.}

\section{Introduction}

Cardiovascular disease (CVD) and associated chronic diseases are widely regarded as the world's leading cause of death $^{1)}$. According to clinical reports, 80 percent of CVDrelated deaths occur in low- and middle-income countries like China ${ }^{2)}$. Atherosclerosis (AS) is a well-known coronary artery disease that causes cardio-cerebrovascular disease, including stroke. Atherosclerosis causes mortality worldwide $^{2,3)}$. According to a few studies, AS is a multifactorial inflammatory disorder characterised by the presence of lesions caused by lipid deposition in the artery walls ${ }^{4}$. At the cellular level, AS is characterised as an excessive inflammatory, proliferative, and fibrofatty response that damages the artery wall and is present in a variety of cell types, including smooth muscle cells (SMC), monocyte-derived macrophages, and endothelial cells $(\mathrm{EC})^{4,5)}$. The development and progression of atherosclerotic plaque is influenced by a number of metabolic, environmental, and genetic factors ${ }^{6,7)}$. Few research suggests that AS can be caused by simple endothelial lining dysfunction, such as that caused by cigarette smoke or hypertension, as well as hyperlipidemia, which causes an excess of nitric oxide (NO) and angiotensin II (Ang II) development in the artery wall $^{4,5,8)}$.

Hypercholesterolemia, a well-known risk factor in humans, such as boosted the level of LDL, TC and other significant contributors to this disease such as insulin resistance, inflammation and oxidative stress ${ }^{9)}$. The expression of adhesion molecules on the endothelium's surface, smooth muscle phenotypic change and proliferation, monocyte invasion of the vessel wall, and the development of foam cells were all triggered as a result of this ${ }^{10,11)}$. The enzyme nitric oxide synthase (eNOS) is essential in the EC. NO derived from eNOS boosts the arterial vasodilatation and reduce the LDL oxidation, platelet aggregation, smooth muscle cell proliferation and adhesion and monocyte adhesion to the endothelium ${ }^{11,12)}$.

Foods are high in cholesterol and saturated fat, both of which have been related to increased circulating cholesterol levels. In the murine model of atherosclerosis, lipid-rich diets are widely used to induce or increase the incidence of atherosclerotic lesions ${ }^{11-13)}$. However, studying atherosclerosis induced by a high-fat diet will help researchers better understand the condition and its treatment effects. The oxidative and lipoprotein oxidation processes are essential in the pathogenesis of atherosclerosis. The increase in lipoprotein fractions and plasma lipids indicates a change in lipid metabolism, which leads to $\mathrm{CVD}^{9,14,15)}$. Last three decades, the researcher focuses their research on the pathological mechanisms and risk factors of $\mathrm{AS}^{2,13)}$. Despite this study, treatments to avoid or, more specifically, reverse the debilitating effects of AS are still a long way off.

It is well documented that hyperlipidemia, inflammation and oxidative stress play an important role in the progres- sion and expansion of AS disease, but still the pathogenesis factor function of AS still unknown ${ }^{11,16)}$. The currently available treatment for AS is antihypertensive and lipid regulating drugs. Moreover, since the atherogenesis mechanism is very complex, high doses and long-term therapy are required ${ }^{17,18)}$. Simvastatin is commonly used drug for treating AS, but this treatment has side effects such as hepatotoxicity and myopathy. Furthermore, few studies suggest that the combination therapy is more effective for treating AS.

Natural substances have become increasingly popular as antioxidants and functional nutrients recently. Crocetin (CT), a carotenoid phytoconstituent isolated from the saffron (Crocus sativa) or Gardenia jasminoides Ellis ${ }^{19,20)}$. Crocetin commonly used as natural food colorant and spice in various parts of the world. Additionally, gardenia and saffron fruits have been mostly used as traditional medicines. Recent years, crocetin showed antioxidant effects to counter the free radicals or oxidative stress and demonstrated anti-inflammatory effects on various animal models ${ }^{21,22)}$. CT exert various types of pharmacological effects such as anti-apoptotic, anticancer, heart disease, neuroprotective, and anti-inflammatory effects ${ }^{19,20,23,24)}$. This effect of CT, especially its capacity to suppress inflammation and oxidative stress, proposes that might be an important candidate to attenuate atherosclerosis. Still, the specific role of crocetin in the prevention of atherosclerosis remains unclear. Consequently, in this experimental study, we explored the anti-atherosclerosis effect of crocetin on high fat induced atherosclerosis in rats and explore the underlying mechanism.

\section{Material and Methods \\ 2.1 Chemicals}

Crocetin (98\%) and simvastatin were purchased from Hangzhou Merck Pharmaceutical Co., Ltd, China. Vitamin D3 was purchased from Shanghai General Pharmaceutical Co., Ltd. Malondialdehyde (MDA), lactate dehydrogenase $(\mathrm{LDH})$, superoxide dismutase (SOD) and nitric oxide (NO) was purchased from the Jincheng Bioengineering Institute, Nanjing, China. Lipid parameters such as TC, LDL, HDL and TG were estimated using the standard available kits (Beijing Beihua Kangtai Clinical Reagent Company Ltd. China). C-reactive protein (hs-CRP) (Wuhan Uscn Co., Ltd), endothelin 1(ET-1), thromboxane B2(TXB2), cardiac troponin $\mathrm{I}(\mathrm{cTnI})$, creatine kinase isoenzyme (CK-MB) kits were purchased from the Wuhan CUSABIO Co., Ltd. China.

\subsection{Animal}

The experimental study used pathogen-free SpragueDawley (SD) rats (aged 8-10 weeks; 200-30 g; both sexes). 
The rats were obtained from the Institutional Animal House and kept in normal laboratory conditions, which included a temperature of $22^{\circ} \mathrm{C}$ and a relative humidity of $65-70 \%$. The rats were kept in the $12 \mathrm{~h}$ light/dark cycle and were received standard laboratory rat chow and tap water (ad libitum). All of the experiments were carried out in accordance with the Guide for the Care and Use of Laboratory Animals and other relevant ethical guidelines. This research was approved by Shandong Xinzhonglu Hospital of Traditional Chinese Medicine animal ethical committee(SDXHTC2021001).

\subsection{High fat composition}

High fat contains cholesterol (3\%), sugar (5\%), propylthiouracil $(0.2 \%)$, sodium cholate $(0.5 \%)$, lard $(10 \%)$ and basic feed $(81.3 \%)$ obtained from the Medical Science Experimental Animal Center, Guangdoung, China.

\subsection{Experimental protocol}

After a week of acclimatisation and adaptive feeding, the rats were released. The rats were divided into the groups below, each of which contains 12 rats.

Group I: a control group received the standard diet and an equal volume of saline,

Group II: The atherosclerotic group received an equal volume of saline,

Group III: atherosclerotic group received the crocetin(5 $\mathrm{mg} / \mathrm{kg})$,

Group IV: atherosclerotic group received the crocetin (10 $\mathrm{mg} / \mathrm{kg}$ ),

Group V: atherosclerotic group received the crocetin(15 $\mathrm{mg} / \mathrm{kg}$ ) and

Group VI: The atherosclerotic group received simvastatin $(0.5 \mathrm{mg} / \mathrm{kg})$, respectively.

Atherosclerosis was caused by combining a high fat diet with a vitamin D3 supplement. Each group of rats was fed a high-fat (15 g) diet once a day. The normal control group rats received the basic diet $(15 \mathrm{~g})$. High fat administration (on the $1^{\text {st }}$ day), control and atherosclerotic group rats were received an equal concentration of normal saline and vitamin D3 (600,000 IU/kg) using normal saline and intraperitoneal injection ${ }^{2)}$. Drugs were administered intragastrically to all of the rats in both the test and control groups. Drug dosages were given to all of the rats in each category based on their weight. Natural saline was administered intragastrically to the normal control and atherosclerotic groups of rats. All of the drug treatments provided to the animal were prepared in the form of a suspension by dissolving a suitable amount of the drug in regular saline solution right before administration.

\subsection{Sample collection}

After successfully anaesthetizing rats with chloral hydrate, blood samples from the abdominal aorta of the rats were obtained and stored in a vacuum tube without the use of an anticoagulant.

\subsection{Biochemical parameters}

Biochemical parameters such as high-sensitivity C-reactive protein (hs-CRP), lactate dehydrogenase (LDH), creatine kinase myocardial bound (CK-MB), and Thromboxane B2 (TXB2) were estimated using the available kits following the manufacture protocol(Wuhan CUSABIO Co., Ltd. China).

In the liver, the HMG-CoA/mevalonate ratio, total protein, and collagen were measured using the previously described method with minor modifications. The level of free fatty acids (FFA) in the serum was calculated using a UV-visible spectrophotometer and a colour reagent.

\subsection{Antioxidant parameter}

Antioxidant parameters such as CAT, GSH, MDA, GPx and SOD were estimated using the available kits following the manufacture instruction (Jincheng Bioengineering Institute, Nanjing, China).

\subsection{Hepatic parameters}

Hepatic parameters such as aspartate aminotransferase (AST), alanine aminotransferase (ALT) and alkaline phosphatase (ALP) were estimated using the available kits following the manufacture instruction ( $R$ \& D Systems, Minneapolis, MN, USA).

\subsection{Lipid parameters}

The available kits were used to estimate lipid parameters such as HDL, LDL, TG, and TC according to the manufacturer's instructions (Beijing Beihua Kangtai Clinical Reagent Company Ltd. China). Using the formulas below, the atherogenic index and coronary risk factor were calculated.

Atherogenic index $=\mathrm{LDL} / \mathrm{HDL}$

Coronary risk index $=\log 10(\mathrm{TG} / \mathrm{HDL})$

\subsection{Inflammatory cytokines and mediators}

Pro-inflammatory cytokines include interleukin- $1 \beta$ (IL-1 $\beta$ ), interleukin-6 (IL-6) and tumor necrosis factor- $\alpha$ (TNF- $\alpha$ ) were estimated using the available kits following the manufacture instruction(R \& D Systems, Minneapolis, MN, USA).

\subsection{Reverse transcription polymerase chain reaction (RT-PCR)}

Complete RNA was isolated from aortic tissue using the TRIzol Reagent. The RNA was reverse-transcribed to cDNA using the RevertAid First Strand cDNA Synthesis Kit. With RT 2 SYBR Green Master and Quantitative SYBR Green 
L. Yu, Q. Kong, R. Gao et al.

Table 1 List of primers.

\begin{tabular}{cccc}
\hline \multirow{2}{*}{ S. No } & \multirow{2}{*}{ Primer } & \multicolumn{2}{c}{ Sequence (5'-3') } \\
\cline { 3 - 4 } & & Forwarded & Reverse \\
\hline 1 & ICAM-1 & CAGTGA CCATCTACAGCTTTCCGG & GCTGCTACCACAGTGATGACAA \\
2 & VCAM-1 & CCCTTGACCGGCTGGAGATT & TGGGGGCAACATTGACATAAAGTG \\
3 & MCP-1 & CCCCAGTCACCTGCTGTTAT & CCACAATGGTCTTGAAGATCAC \\
4 & GAPDH & ACGGATTTGGTCGTATTGGG & TGATTTTGGAGGGATCTCGC \\
\hline
\end{tabular}

Table 2 The effect on the body weight in different experimental groups of rats.

\begin{tabular}{ccccccc}
\hline \multirow{2}{*}{ S. No } & \multirow{2}{*}{ Groups } & \multicolumn{5}{c}{ Days } \\
\cline { 3 - 7 } & & \multicolumn{1}{c}{ Day 1 } & \multicolumn{1}{c}{ Day 7 } & Day 14 & Day 21 & Day 30 \\
\hline 1 & Group I & $115.4 \pm 7.45$ & $120.3 \pm 6.54$ & $129.2 \pm 5.93$ & $138.4 \pm 6.49$ & $145.6 \pm 6.82$ \\
2 & Group II & $121.3 \pm 8.59$ & $128.3 \pm 6.32$ & $141.8 \pm 6.91$ & $152.9 \pm 7.34$ & $169.2 \pm 9.74$ \\
3 & Group III & $116.8 \pm 6.54^{\text {ns }}$ & $125.3 \pm 6.49^{\text {ns }}$ & $137.3 \pm 7.34^{*}$ & $148.8 \pm 6.34^{*}$ & $164.5 \pm 8.45^{*}$ \\
4 & Group IV & $115.2 \pm 7.93^{\text {ns }}$ & $123.3 \pm 5.94^{*}$ & $131.4 \pm 6.92^{* *}$ & $145.3 \pm 5.34^{* *}$ & $160.3 \pm 7.34^{* *}$ \\
5 & Group V & $116.2 \pm 9.23^{\text {ns }}$ & $121.3 \pm 4.53^{*}$ & $130.8 \pm 6.03^{* * *}$ & $139.8 \pm 5.89^{* * *}$ & $148.1 \pm 5.39^{* * *}$ \\
6 & Group VI & $116.5 \pm 7.46^{\text {ns }}$ & $122.4 \pm 4.81^{*}$ & $131 \pm 6.15^{* * *}$ & $140.3 \pm 5.91^{* * *}$ & $148.9 \pm 5.83^{* * *}$ \\
\hline
\end{tabular}

Data are compared with the atherosclerosis control group rats. Where $* p<0.05$ was significant, $* * p<0.01$ more significant and $* * * p<0.001$ extreme significant.

PCR assays, the primers (Table 1) were used to determine gene expression. All samples were subjected to a $98^{\circ} \mathrm{C}$ for 2 min program, followed by 25 to 40 cycles of $98^{\circ} \mathrm{C}$ for 10 sec, $55^{\circ} \mathrm{C}$ for $5 \mathrm{sec}$, and $72^{\circ} \mathrm{C}$ for $20 \mathrm{sec}$. The relative standard curves were used to measure the mRNA expression levels. Plotting the quantification period $(\mathrm{Cq})$ against the log sum of total cDNA applied to the reaction yielded the curves. The 2-Cq approach was used to assess the relative target gene expression levels.

\subsection{Statistical analysis}

The Graph Pad Prism Software, version 7, was used to conduct the statistical analysis. All data are presented as a mean with standard deviation (SEM). The Dunnett's test was used to compare the care groups to the disease control and vehicle control groups using analysis of variance (ANOVA). The statistically relevant level was set at $p<$ 0.05 .

\section{Results}

3.1 Effect of crocetin on body weight, organ weight, food intake and calorie intake

During the progression of atherosclerosis, an increase in body weight plays an important role in the disease progression. When compared to the normal control group, the AS control group rats had a higher body weight. Crocetin (15 $\mathrm{mg} / \mathrm{kg}$ ) treated rats exhibited improvement in body weight, similar to the pattern of normal rats. Crocetin (5 and 10 $\mathrm{mg} / \mathrm{kg}$ ) treated rats showed increased body weight but not similar to the normal control group manner (Table 2).

Crocetin effect on organ weight (heart, liver, and kidney) was shown in Table 3. AS control group rats showed the alteration in the heart, liver and kidney weight, which was not significant. The crocetin-treated group rats showed a similar result.

Table 4 exhibited the food and calorie intake of the different groups of rats. AS control group rats showed the reduction in the food intake and enhancement in the calorie intake compared to the normal rats. Crocetin treated group rats showed improvement in food intake and reduction in the calorie intake.

\subsection{Effect of crocetin on aorta medium and platelet ag- gregation rate}

Figure 1a exhibited the effect of crocetin on the aorta medium. During atherosclerosis, aortic medium thickness increases due to the expansion of the disease. Normal control group rats showed a normal manner of thickness of aorta medium. AS control group rats showed increased thickness of the aorta thickness and suggest the expansion of disease. Crocetin treatment significantly $(p<0.001)$ decreased the thickness of aorta medium and suggesting the protective effect against atherosclerosis.

Figure $1 \mathrm{~b}$ demonstrated the increased platelet aggregation rate in the AS group rats and crocetin treated group rats showed the decrease of platelet aggregation rate. 
Table 3 The effect of the organ weight in different experimental groups of rats.

\begin{tabular}{ccclc}
\hline \multirow{2}{*}{ S. No } & \multirow{2}{*}{ Groups } & \multicolumn{3}{c}{ Organ weight $(\mathrm{g})$} \\
\cline { 3 - 5 } & & \multicolumn{1}{c}{ Heart } & \multicolumn{1}{c}{ Liver } & Kidney \\
\hline 1 & Group I & $0.802 \pm 0.020$ & $7.41 \pm 0.63$ & $2.21 \pm 0.12$ \\
2 & Group II & $0.756 \pm 0.018$ & $8.38 \pm 0.78$ & $2.23 \pm 0.13$ \\
3 & Group III & $0.759 \pm 0.019^{\text {ns }}$ & $8.21 \pm 0.83^{*}$ & $2.24 \pm 0.11^{\text {ns }}$ \\
4 & Group IV & $0.783 \pm 0.021^{\text {ns }}$ & $7.98 \pm 0.68^{* *}$ & $2.23 \pm 0.12^{\text {ns }}$ \\
5 & Group V & $0.801 \pm 0.23^{*}$ & $7.65 \pm 0.72^{* * *}$ & $2.2 \pm 0.14^{\text {ns }}$ \\
6 & Group VI & $0.800 \pm 0.22^{\text {ns }}$ & $7.72 \pm 0.69^{* * *}$ & $2.21 \pm 0.12^{\text {ns }}$ \\
\hline
\end{tabular}

Data are compared with the atherosclerosis control group rats. Where $* p<0.05$ was significant, $* * p<0.01$ more significant and $* * * p<0.001$ extreme significant.

Table 4 The effect on the food and calorie of different experimental groups of rats.

\begin{tabular}{cccc}
\hline \multirow{2}{*}{ S. No } & \multirow{2}{*}{ Groups } & \multicolumn{2}{c}{ Food and calorie } \\
\cline { 3 - 4 } & & Food intake $(\mathrm{g} /$ day $)$ & Calorie intake $(\mathrm{kcal} /$ day $)$ \\
\hline 1 & Group I & $18.34 \pm 3.24$ & $60.4 \pm 2.83$ \\
2 & Group II & $17.52 \pm 2.89$ & $69.9 \pm 3.96$ \\
3 & Group III & $17.84 \pm 3.03^{\text {ns }}$ & $68.3 \pm 3.88^{\text {ns }}$ \\
4 & Group IV & $18.04 \pm 2.86^{*}$ & $66.9 \pm 4.02^{*}$ \\
5 & Group V & $18.32 \pm 2.93^{* *}$ & $61.4 \pm 3.94^{* * *}$ \\
6 & Group VI & $18.15 \pm 2.56^{* *}$ & $61.6 \pm 3.36^{* * *}$ \\
\hline
\end{tabular}

Data are compared with the atherosclerosis control group rats. Where $* p<$ 0.05 was significant, ${ }^{* *} p<0.01$ more significant and $* * * p<0.001$ extreme significant.

a

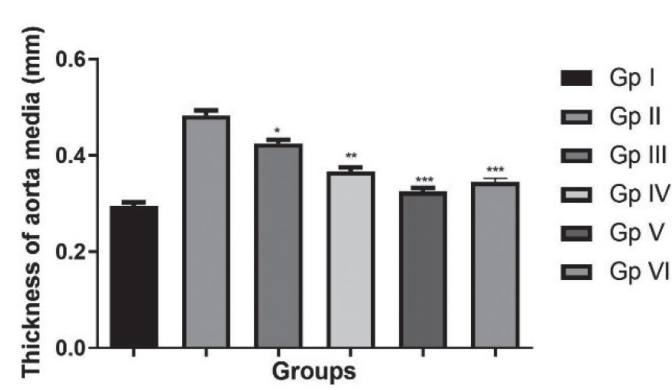

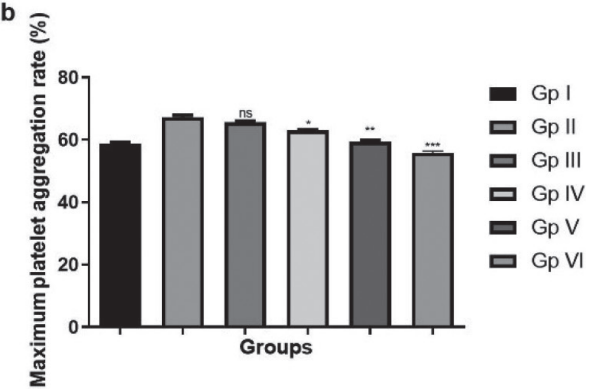

Fig. 1 Effect of crocetin (CT) on the aorta thickness media and maximum platelet aggregation rates in High diet and vitamin D induced atherosclerosis $(\mathrm{AS})$ in rats. a: thickness of aorta media and $\mathbf{b}$ : maximum platelet aggregation. Data are compared with the atherosclerosis control group rats. Where ${ }^{*} p<0.05$ was significant, ${ }^{* *} p<0.01$ more significant and $* * * p<0.001$ extreme significant.

\subsection{Effect of crocetin on lipid parameters}

Figure 2 showed that the crocetin altered lipid parameters. During atherosclerosis, altered lipid parameters are common problems. AS group rats showed increased levels of VLDL (Fig. 2a), LDL (Fig. 2b), TG (Fig. 2d), TC (Fig. 2e) and reduced the level of HDL (Fig. 2c). Crocetin treatment significantly $(p<0.001)$ suppressed the level of VLDL, LDL, TG, TC and improved the level of HDL.
During heart disease, especially atherosclerosis, the atherogenic index and coronary risk factors rise. In this experiment, the AS group rats had higher levels of atherogenic index and coronary risk factor than the control group. Crocetin treatment significantly $(p<0.001)$ reduced the level of atherogenic index (Fig. 3a) and coronary risk factor (Fig. 3b).

We also estimated the ratio of lipid parameters such as 
a
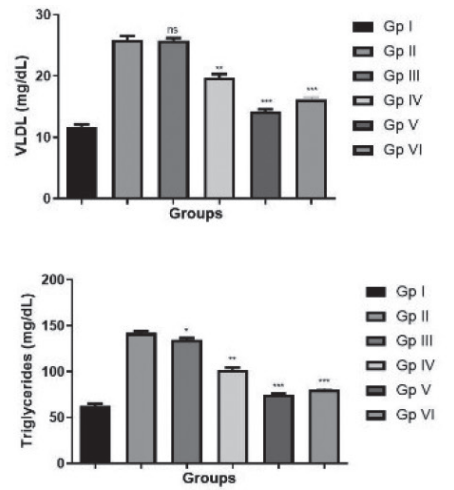

b

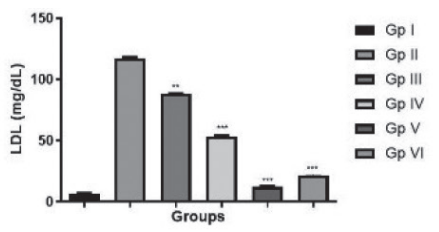

e

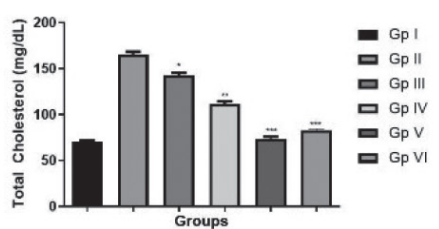

c

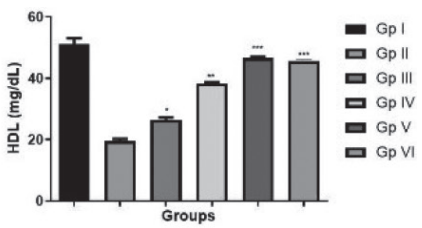

Fig. 2 Effect of crocetin(CT) on the lipid parameters in High diet and vitamin D induced atherosclerosis (AS) in rats. a: VLDL, b: LDL, c: HDL, d: triglyceride and e: total cholesterol. Data are compared with the atherosclerosis control group rats. Where $* p<0.05$ was significant, $* * p<0.01$ more significant and $* * * p<0.001$ extreme significant.
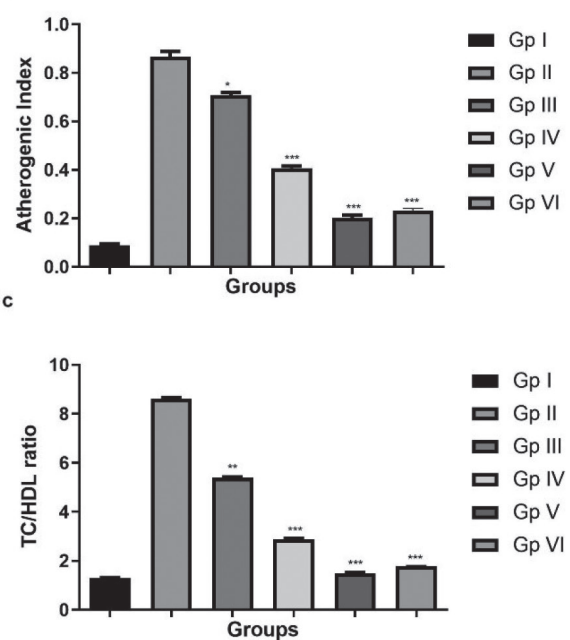
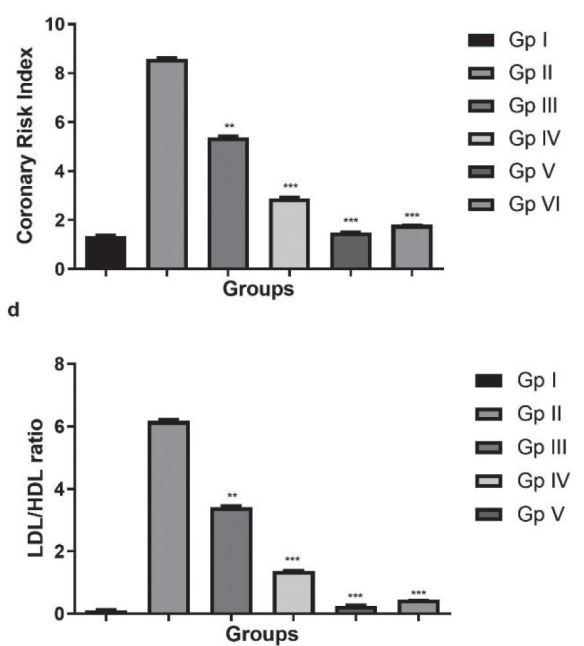

Fig. 3 Effect of crocetin (CT) on the atherogenic index, coronary risk factor, TC/HDL and LDL/HDL in High diet and vitamin D induced atherosclerosis (AS) in rats. a: atherogenic index, b: coronary risk factor, $\mathbf{c}$ : TC/HDL and d: LDL/HDL. Data are compared with the atherosclerosis control group rats. Where $* p<0.05$ was significant, ${ }^{* *} p<0.01$ more significant and $* * * p<0.001$ extreme significant.

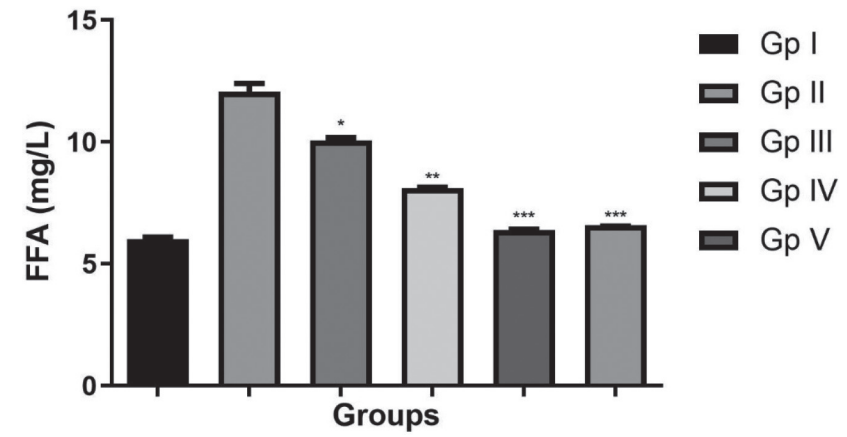

Fig. 4 Effect of crocetin (CT) on the free fatty acid in High diet and vitamin D induced atherosclerosis(AS) in rats. Data are compared with the atherosclerosis control group rats. Where $* p<0.05$ was significant, $* * p<0.01$ more significant and $* * * p<0.001$ extreme significant.
TC/HDL and LDL/HDL in this experimental study. The ratio of TC/HDL (Fig. 3c) and LDL/HDL (Fig. 3d) boosted in the AS group and crocetin treatment significantly $(p<$ 0.001 ) reduced the ratio of TC/HDL and LDL/HDL.

\subsection{Effect of crocetin on free fatty acid (FFA)}

During atherosclerosis, the level of FFA boosted due to alteration of lipid profile and expansion of disease. Figure 4 showed the increased level of FFA in the AS group and crocetin treatment significantly $(p<0.001)$ reduced the FFA level.

\subsection{Effect of crocetin on serum hs-CRP, NO, TXB2 and ET-1}

The activities of hs-CRP (Fig. 5a), TXB2 (Fig. 5b), NO (Fig. 5c) and ET-1 (Fig. 5d)were in the AS group rats. Crocetin treatment significantly $(p<0.001)$ dose-dependently 
a

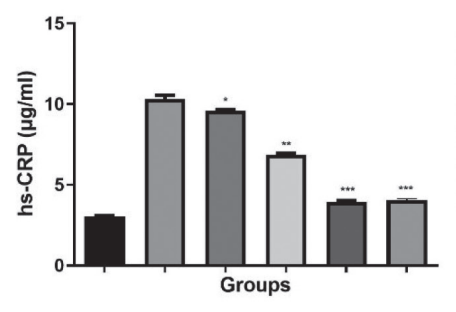

c

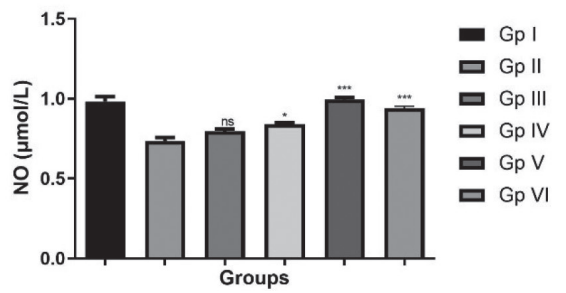

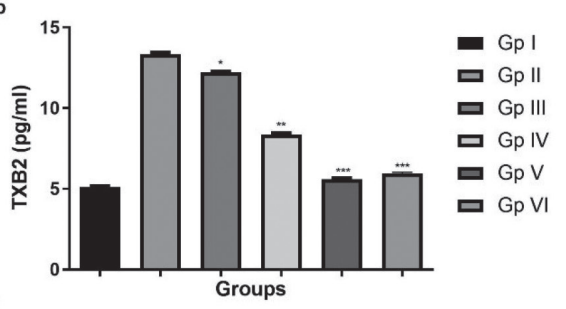

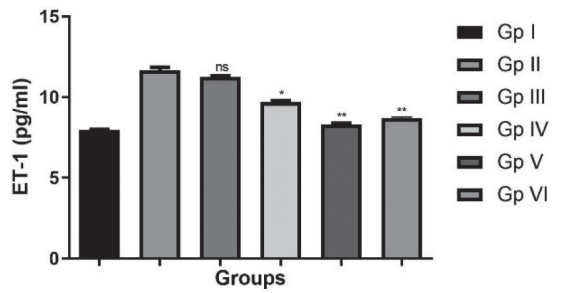

Fig. 5 Effect of crocetin (CT) on the hs-CRP, TXB2, NO and ET-1 in High diet and vitamin D induced atherosclerosis (AS) in rats. a: atherogenic index, b: coronary risk factor, $\mathbf{c}$ : TC/HDL and $\mathbf{d}$ : LDL/HDL. Data are compared with the atherosclerosis control group rats. Where $* p<0.05$ was significant, $* * p<0.01$ more significant and $* * * p<0.001$ extreme significant.

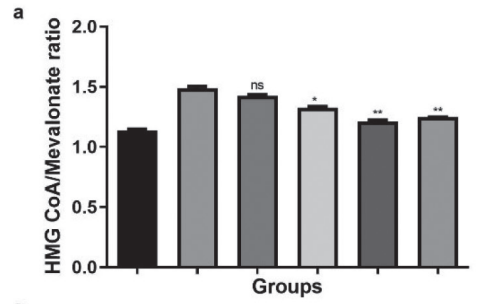

c

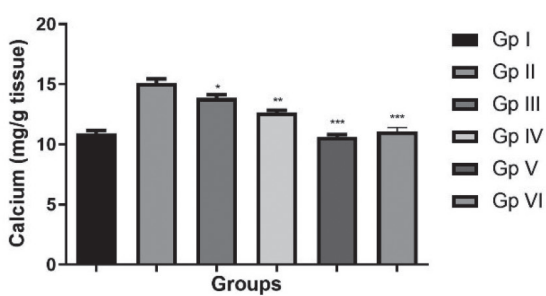

b

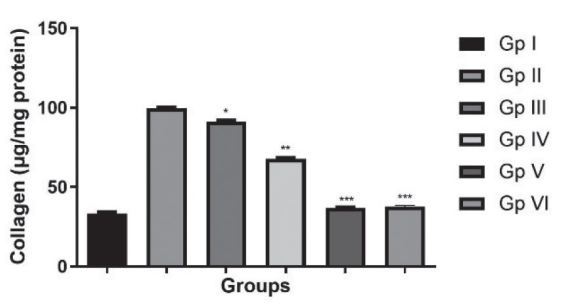

d

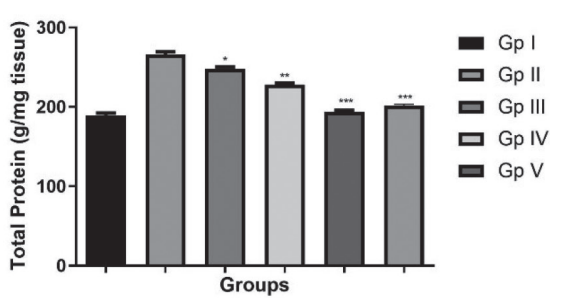

Fig. 6 Effect of crocetin (CT) on the HMG-CoA/Mevalonate ratio, collagen, calcium and total protein in High diet and vitamin D induced atherosclerosis $(\mathrm{AS})$ in rats. a: HMG-CoA/Mevalonate ratio, b: collagen, c: calcium and d: total protein. Data are compared with the atherosclerosis control group rats. Where $* p<0.05$ was significant, $* * p<0.01$ more significant and ${ }^{* * *} p<0.001$ extreme significant.

reduced the activities of hs-CRP(Fig. 5a), TXB2 (Fig. 5b), NO (Fig. 5c) and ET-1 (Fig. 5d).

\subsection{Effect of crocetin on collagen, HMG-CoA reductase, calcium and total protein}

Figure 6 showed the effect of collagen, total protein, HMG-CoA reductase and calcium. AS group rats showed increased levels of HMG-CoA reductase(Fig. 6a), collagen (Fig. 6b), calcium (Fig. 6c) and total protein (Fig. 6d) compared to the normal group. AS group rats treated with the crocetin significantly $(p<0.001)$ reduced the levels of collagen, HMG-CoA reductase, total protein and calcium and suggest the anti-atherosclerosis effect.

\subsection{Effect of crocetin on antioxidant parameters}

Table 5 showed the effect of crocetin on the antioxidant parameters of AS rats. AS group rats exhibited the increased level of MDA and reduced level of GSH, SOD, GPx and CAT as compared to normal rats. Crocetin treatment significantly $(p<0.001)$ down-regulated the MDA level and improved the GSH, SOD, GPx and CAT.

\subsection{Effect of crocetin on cardiac parameters}

Cardiac parameters such as LDH, cTnl and CK-MB boosted during cardiac diseases such as atherosclerosis. Figure 7 showed increased levels of $\mathrm{LDH}$ (Fig. 7a), cTnl (Fig. 7b) and CK-MB (Fig. 7c) in the AS control group rats 
Table 5 The effect of crocetin on the antioxidant parameters in different experimental groups of rats.

\begin{tabular}{ccccccc}
\hline \multirow{2}{*}{ S. No } & \multirow{2}{*}{ Groups } & \multicolumn{5}{c}{ Antioxidant parameters } \\
\cline { 3 - 7 } & & MDA & SOD & CAT & GSH & GPx \\
\hline 1 & Group I & $2.05 \pm 0.28$ & $143.4 \pm 6.94$ & $110.7 \pm 4.03$ & $17.9 \pm 1.93$ & $2.03 \pm 0.65$ \\
2 & Group II & $10.34 \pm 1.23$ & $58.7 \pm 6.32$ & $60.2 \pm 3.21$ & $9.34 \pm 1.14$ & $0.45 \pm 0.11$ \\
3 & Group III & $8.31 \pm 1.12^{*}$ & $68.4 \pm 5.73^{*}$ & $70.5 \pm 3.82^{* *}$ & $11.3 \pm 1.03 *$ & $0.74 \pm 0.14 * *$ \\
4 & Group IV & $6.49 \pm 1.02 * *$ & $94.1 \pm 5.96 * *$ & $85 \pm 3.71 * * *$ & $13.6 \pm 1.87 * *$ & $1.02 \pm 0.38^{* * *}$ \\
5 & Group V & $2.56 \pm 0.84 * * *$ & $136.3 \pm 6.39 * * *$ & $105.3 \pm 3.91 * * *$ & $17.5 \pm 1.34 * * *$ & $1.96 \pm 0.49 * * *$ \\
6 & Group VI & $2.91 \pm 1.02^{* * * *}$ & $134.2 \pm 5.91 * * *$ & $102.5 \pm 2.83^{* * *}$ & $17.3 \pm 1.12^{* * *}$ & $1.90 \pm 0.43^{* * *}$ \\
\hline
\end{tabular}

Data are compared with the atherosclerosis control group rats. Where ${ }^{*} p<0.05$ was significant, ${ }^{* *} p<0.01$ more significant and ${ }^{* * *} p<0.001$ extreme significant. MDA (nmol/mg protein), SOD (unit/mg protein), CAT (unit $/ \mathrm{mg}$ protein), GST (unit/ mg protein), GSH (nmol GSH Eq./mg protein)
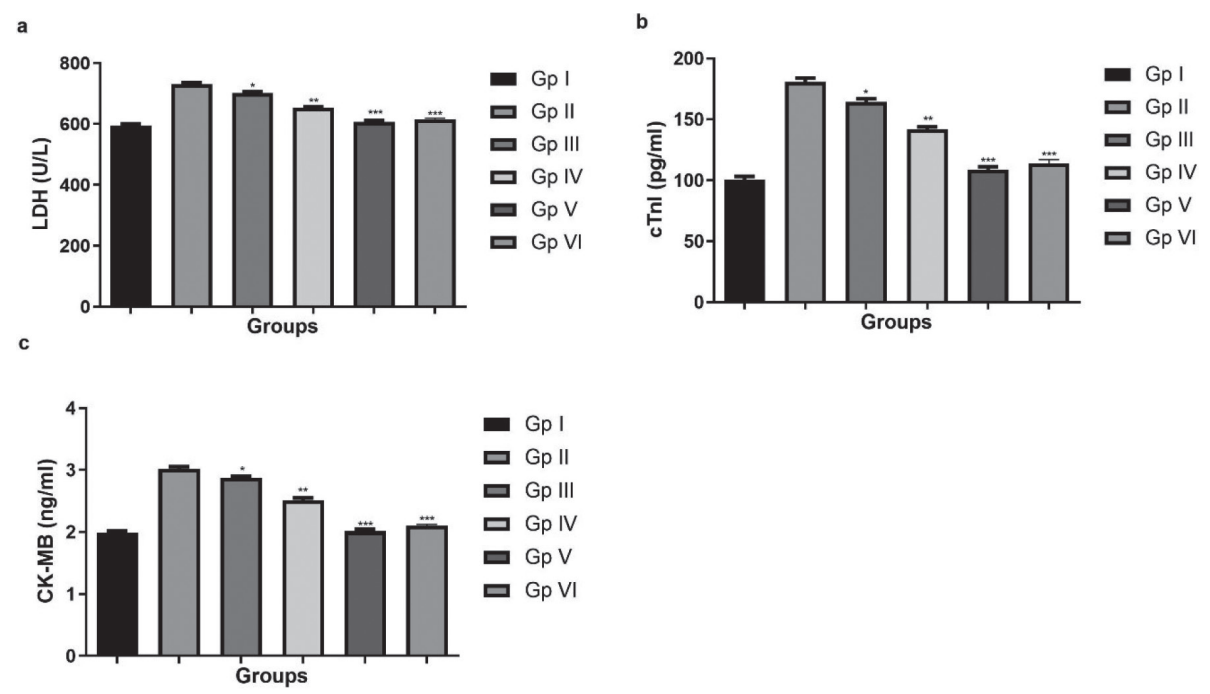

Fig. 7 Effect of crocetin(CT) on the heart parameters in High diet and vitamin D induced atherosclerosis (AS) in rats. a: $\mathrm{LDH}, \mathbf{b}$ : cTnl and $\mathbf{c}$ : CK-MB. Data are compared with the atherosclerosis control group rats. Where $* p<0.05$ was significant, ${ }^{* *} p<0.01$ more significant and ${ }^{* * *} p<0.001$ extreme significant.

and crocetin treatment significantly $(p<0.001)$ suppressed the level.

\subsection{Effect of crocetin on hepatic parameters}

Crocetin effect on hepatic parameters in atherosclerosis rats was shown in Fig. 8. When compared to standard rats, AS control rats had higher levels of hepatic parameters like $\operatorname{AST}$ (Fig. 8a), ALT(Fig. 8b), and ALP(Fig. 8c). Crocetin treatment significantly $(p<0.001)$ reduced the level of AST, ALT and ALP.

\subsection{Effect of crocetin on inflammatory cytokines and me- diators}

Crocetin effect on the levels of inflammatory cytokines and mediators was shown in Fig. 9. Increases the inflammatory response, which stimulates the release of inflammatory cytokines and mediators during atherosclerosis disease. A similar result was observed in this experimental study, AS group rats showed that the increased levels of inflammatory cytokines include TNF- $\alpha$, IL-1 $\beta$, IL-6 (Fig. 9a-9c) and inflammatory mediators NF-kB (Fig. 9d). Crocetin treatment decreased the level of inflammatory cytokines includes TNF- $\alpha$ (Fig. 9a), IL-1 $\beta$ (Fig. 9b), IL-6 (Fig. 9c) and inflammatory mediators NF-кB (Fig. 9d) compared with the AS group.

\subsection{Effect of crocetin on mRNA expression}

AS group showed the increased expression of VCAM-1 (Fig. 10a), ICAM-1 (Fig. 10b) and MCP-1 (Fig. 10c) as compared to control group. Crocetin treated group rats significantly $(p<0.001)$ suppressed the mRNA expression.

\section{Discussion}

Myocardial infarction is usually diagnosed using hepatic 
a

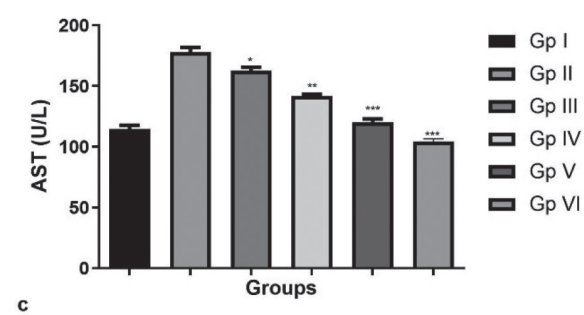

c

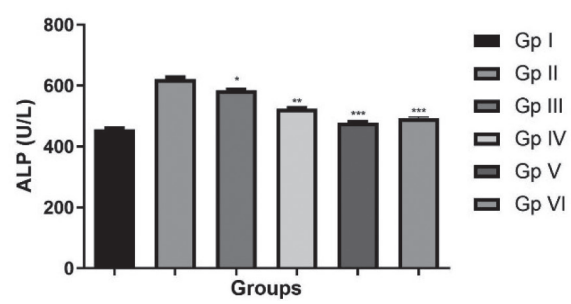

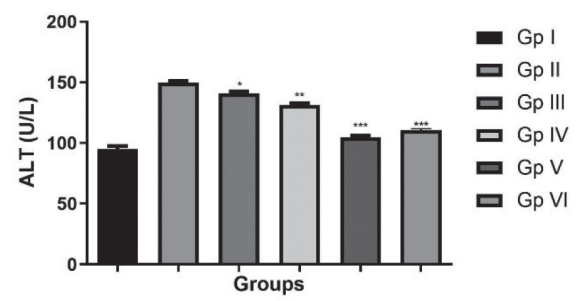

Fig. 8 Effect of crocetin(CT) on the hepatic parameters in High diet and vitamin D induced atherosclerosis (AS) in rats. a: AST, b: ALT and c: ALP. Data are compared with the atherosclerosis control group rats. Where $* p<0.05$ was significant, ${ }^{* *} p<0.01$ more significant and ${ }^{* * *} p<0.001$ extreme significant.

a

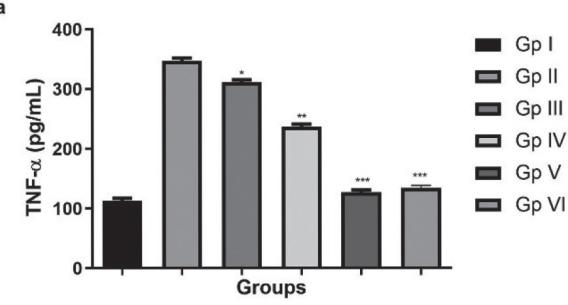

c

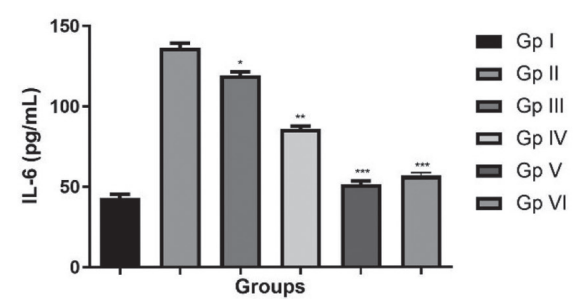

b

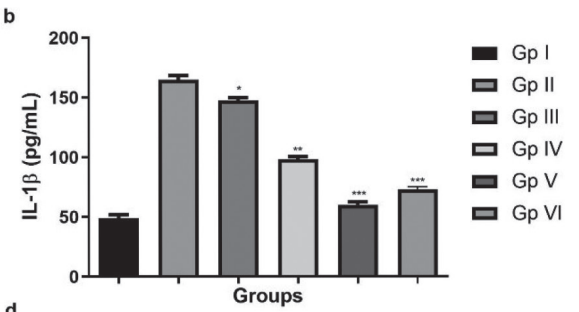

d

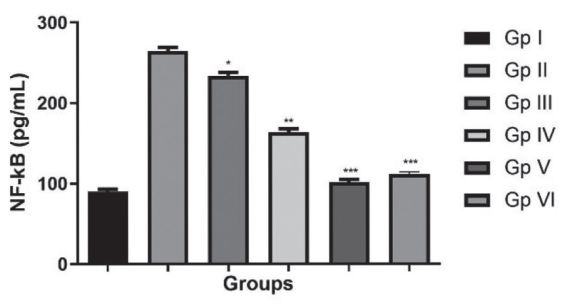

Fig. 9 Effect of crocetin(CT) on the inflammatory cytokines and inflammatory parameters in High diet and vitamin D induced atherosclerosis (AS) in rats. a: TNF- $\alpha, \mathbf{b}$ : IL-1 $\beta$, c: IL-6 and d: NF- $\mathrm{kB}$. Data are compared with the atherosclerosis control group rats. Where ${ }^{*} p<0.05$ was significant, $* * p<0.01$ more significant and $* * * p<0.001$ extreme significant.

parameters. For myocardial infarction, hepatic parameters such as AST are widely used as diagnostic markers ${ }^{4)}$. Enzymes such as AST, LDH, ALT, and CPK leak from necrotic heart cells into the bloodstream during pathological conditions, and these enzymes are important estimating markers of cardiac injury ${ }^{4}$. These enzymes are not specific for myocardial injury on their own; however, a combination of these enzymes can indicate myocardial damage. CPK, another important enzyme in the body's energy metabolism, maintains a high level of intracellular ATP through phosphorylation and is thought to be a good indicator of atherosclerosis. AS rats had higher levels of AST, LDH, ALT, and CPK, which were greatly decreased by crocetin treatment.

Hypercholesteremia is one of the significant factors in the expansion of atherosclerosis and high fat diet induced atherosclerotic model in rats widely used model ${ }^{25,26)}$. The current investigation result showed that the crocetin decrease the lipid concentration in the serum of atherosclerotic rats. During the hypercholesterolemic rats, the lipid parameters and inflammatory mediators boosted in the serum. Various factors, such as uncontrolled oxidative stress and the inflammatory response, play a significant role in the progression of atherosclerosis. Investigations suggest that the inflammatory cytokines activate macrophages and cause the proliferation of endothelial cells ${ }^{16)}$. 
a

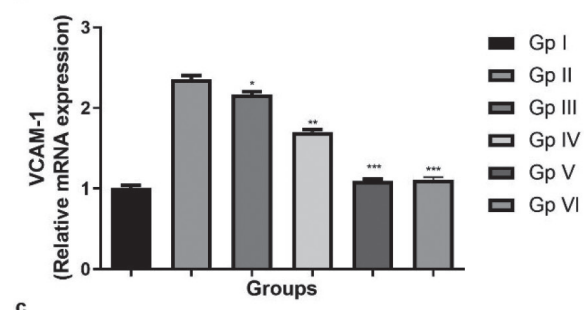

c

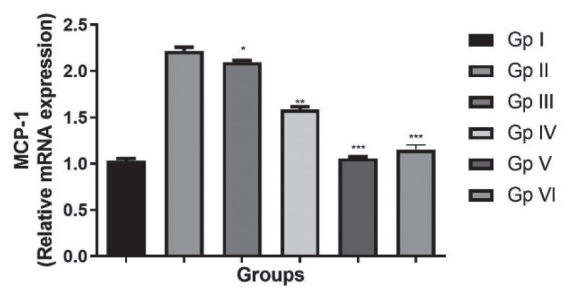

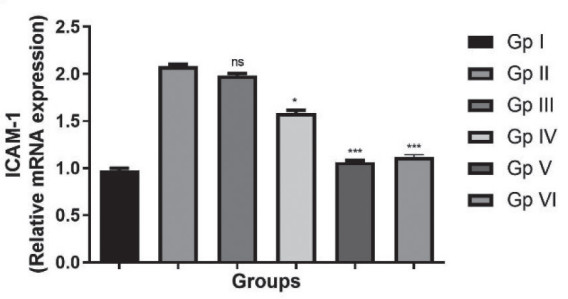

Fig. 10 Effect of crocetin (CT) on the mRNA expression in High diet and vitamin D induced atherosclerosis (AS) in rats. a: VCAM-1, b: ICAM-1 and c: MCP-1. Data are compared with the atherosclerosis control group rats. Where $* p<0.05$ was significant, ${ }^{* *} p<0.01$ more significant and ${ }^{* * *} p<0.001$ extreme significant.

Additionally, it has been reported that the expansion of atherosclerosis is managed by few anti-inflammatory drugs.

Dyslipidamia increases the accumulation of oxidised LDL in the arterial wall, which is a significant risk factor for coronary artery disease such as atherosclerosis. Oxidized LDL plays a significant role in the progression and initiation of cardiovascular dysfunction related to atherogenesis or atherosclerosis; later amelioration of oxidative stress is equally important in reducing or controlling the dyslipidamia ${ }^{2,27)}$. The increase in oxidative stress plays a significant role in atherogenesis. Oxidative alteration of LDL is deemed an initial step in the conversion of LDL to atherogenic form. Previous research showed the correlation between atherogenic lipoproteins and plasma levels of oxidants in patients is affected by the cardiovascular disease ${ }^{9-11)}$. Atherosclerosis is related to high levels of TC, TG, HDL and reduced levels of HDL in the serum.

Total body cholesterol and HDLc are inversely related, and lowering plasma HDL levels can hasten the expansion and progression of atherosclerosis, which causes ischemia by impairing cholesterol clearance from the arterial wall. Inverse cholesterol transfer, in which excess tissue cholesterol is taken up and processed by the HDL particle before being delivered to the liver for metabolism, is widely attributed to HDL ${ }^{9,11,12)}$. Moreover, the boost the level of HDL help in lowering the atherosclerosis risk. In this experimental study, AS group rats showed reduced levels of HDL and crocetin treatment significantly improved the HDL level and suggesting the anti-hyperlipidemic and atherogenic protective effect.

Much evidence suggests that LDL oxidation is critical in the early stages of atherosclerosis, and that thrombosis is one of the disease's fatal clinical conditions. Inequalities in lipid metabolism also contribute to lipid peroxidation, which was found in the current research. It is well known that having high levels of VDL and LDL raises the risk of coronary heart disease ${ }^{9,13)}$. There is a direct correlation between LDL levels and atherosclerosis, and lowering serum LDL can reverse related pathological events. LDL levels were higher in the AS group rats, and crocetin treatment greatly decreased LDL levels, implying defence against coronary artery disease. The enzyme lecithin cholesterol-O-acyltransferase (LCAT) is involved in the maturation of HDL, cholesterol trans-esterification, and cholesterol flux from cell membranes through $\mathrm{HDL}^{9,28)}$. Crocetin can help to raise HDL levels, which are linked to cholesterol metabolism from peripheral cells to the liver through the LCAT enzymes, which play a key role in lipoprotein metabolism. AS rats had a higher atherogenic index and coronary risk index, while crocetin-treated rats had a slightly lower atherogenic and coronary risk index. Crocetin treatment significantly reduced the TC/HDL and LDL/HDL ratios in the AS group rats, suggesting an anti-hyperlipidemic effect.

It is well documented that vascular inflammatory and oxidative stress boosted during atherosclerosis disease ${ }^{29)}$. Atherosclerosis disease and endothelial damage are exacerbated by vascular inflammatory reactions and oxidative stress. The progression of atherosclerosis disease is influenced by oxidative stress parameters. MDA (a lipid peroxidation marker) is a well-known oxidative stress marker, and its level is often used to estimate oxidative injury ${ }^{4)}$. The high fat induced atherosclerosis in the rats has many common features similar to human atherosclerosis and various investigations have shown that antioxidants act as atherosclerosis in the HFD-induced AS model ${ }^{6,30)}$. Previous investigations suggest that the antioxidant defense system and oxidative stress altered during the atherosclerosis. Atherosclerosis is related to the decreased antioxidant 
defense system in numerous body organs such as the heart tissue; to the current experimental study, AS group rats showed increased levels of MDA and decreased antioxidant enzyme activity. Various investigation suggests that during atherosclerosis boosted the level of oxidative stress in the serum and tissue ${ }^{5,31)}$. Antioxidant enzymes such as GPx, CAT, and SOD are essential in scavenging free radicals or oxygen species and preventing their formation. CAT, GPx and SOD are the chief scavenger of anion superoxide $\left(\mathrm{O}_{2}{ }^{-}\right)$, and during the atherosclerosis the level of CAT, GPx and SOD reduced and crocetin treatment significantly increased the activity of these enzymes ${ }^{5,32}$. The increased level of CAT during the atherosclerosis, may be a significant adaptive response to conditions of boosted oxidative stress in the atherosclerosis and the biological availability of hydrogen peroxide and superoxide anion radicals in the adipose tissue. The boosted level of CAT reflects enhanced hydrogen peroxide and superoxide anion radicals as catalase decomposes hydrogen peroxide to oxygen and water ${ }^{5,6,33)}$. In the current experimental study, crocetin significantly reduced the level of SOD, CAT, GPx and increased level of MDA, showed the antioxidant effects.

The liver is an important organ in which all metabolic reactions continue. The activity of HMG-CoA reductase in liver tissue was measured indirectly using the HMG CoA/ mevalonate ratio. HMG-CoA reductase inhibitors are used to lower serum cholesterol and improve longevity in people who are at risk of atherosclerosis ${ }^{34)}$. Their main mechanism of action is in the liver, where they inhibit HMG-CoA reductase, the enzyme that produces isoprenoids and cholesterol. Crocetin therapy reduced the HMG-CoA/mevalonate ratio significantly, which may be attributed to the inhibition of HMG-CoA reductase activity; therefore, it is clear that the drug has hypolipidemic activity. Crocetin, which competes with HMG-CoA reductase enzymes in the liver to convert HMG-CoA to mevalonate, an early precursor for cholesterol biosynthesis, is one of the most powerful and commonly used medicines for treating hyperlipidemia ${ }^{35,36)}$. As a result, crocetin can suppress the HMG-CoA enzyme, as evidenced by the current study's lipoprotein and lipid status. The distribution of hydroxy proline within collagen can be easily investigated. It's a protein family made up of a variety of genetically distinct molecular species that's essential for tissue function, organisation, growth, and differentiation. When compared to the astescleorsis community, the alterations in the crocetin-treated rats were reversible.

Inflammatory mediators and metabolism disorder commonly observed in the aorta of hypercholesterolemic rats. According to previous research, an unusual inflammatory response can play a significant role in the spread and development of $\mathrm{AS}^{36,37)}$. During atherosclerosis condition, the pro-inflammatory cytokines such as TNF- $\alpha$ and IL- 6 were activated by endothelial cell propagation and activation of macrophages. Therefore, AS group rats showed increased levels of TNF- $\alpha$ and IL- 6 and showed increased inflammatory response ${ }^{16)}$. Crocetin treatment significantly reduced the level of TNF- $\alpha$ and IL- 6 and showed the anti-inflammatory effect of minimization of inflammatory response.

Previous investigation suggests that atherosclerosis not only contributed in the hyperlipidemic condition but also take part in the inflammatory response ${ }^{36)}$. ICAM-1 and VCAM-1 are chemokines that act on adhering to the monocytes into the subendothelial space, which are observed to be different in the endothelium at pro-inflammatory state $^{37)}$. During the higher inflammatory condition, the invaded macrophages tend to release inflammatory cytokines include TNF- $\alpha$ and further activate the inflammatory reaction by activating the formation of foam cells. TNF- $\alpha$ (a pro-inflammatory cytokines), which boosted ICAM-1 and VCAM-1 expression and increases the adherence of monocytes to the endothelial surface ${ }^{38)}$. TNF- $\alpha$ start secretion by macrophages and further activates the secretion the more inflammatory cytokines include IL- 6, IFN- $\gamma$ and IL- $1 \beta$. The expression of adhesion molecules (ICAM-1, MCP-1 and VCAM-1) in the AS group of rats. Previous investigation demonstrated that the increased expression of ICAM-1 and VCAM-1, due to dysfunction of $\mathrm{NO}^{38}$. In this experimental study, we observed that the AS group exhibited an increased level of pro-inflammatory cytokines and adhesion molecules such as ICAM-1 and VCAM- ${ }^{39)}$. Furthermore, in endothelial cells, exhibited the expression of MCP-1 increased due to reduction of NO synthesis. Crocetin treatment significantly reduced the levels of pro-inflammatory cytokines and adhesion molecules expression.

\section{Conclusion}

In this experimental study, we used the high fat diet and vitamin D3 to SD rat model. In this study, crocetin reduced the thickness of aorta medium and platelet aggression rate. Crocetin also altered the lipid profile and reduced the atherogenic index, coronary risk index, TC/HDL and LDL/HDL ratio. Crocetin significantly reduced the level of hs-CRP, TXB2, NO and ET-1. Crocetin resisting the oxidation, inflammation and heart parameters. According to the current research, crocetin maintains lipoprotein and lipid status through the metabolic key enzyme HMG-CoA reductase, as well as calcium and collagen levels, which plays an important role in atherosclerosis. The pharmacological effect of crocetin may significant role in prevention and treatment of serve atherosclerotic cardio-cerebrovascular disease.

\section{Author Contributions}

L.Y., designed and performed research. Q.K., R.G., and X.L., contributed analytic tools; analyzed data. X.S., design 


\section{Yu, Q. Kong, R. Gao et al.}

the study, procured the necessary chemical, kits for the current study and wrote the manuscript. All the authors draft and read the proof of manuscript.

\section{References}

1) Nitsa, A.; Toutouza, M.; Machairas, N.; Mariolis, A.; Philippou, A.; Koutsilieris, M. Vitamin D in cardiovascular disease. In Vivo (Brooklyn) 32, 977-981 (2018).

2) Fu, W.J.; Lei, T.; Yin, Z.; Pan, J.H.; Chai, Y.S.; Xu, X.Y.; Yan, Y.X. et al. Anti-atherosclerosis and cardio-protective effects of the Angong Niuhuang Pill on a high fat and vitamin D3 induced rodent model of atherosclerosis. J. Ethnopharmacol. 195, 118-126(2017).

3) Wen, S.Y.; Tamilselvi, S.; Shen, C.Y.; Day, C.H.; Chun, L.C.; Cheng, L.Y.; Ou, H.C.; Chen, R.J. et al. Protective effect of HDL on NADPH oxidase-derived super oxide anion mediates hypoxia-induced cardiomyocyte apoptosis. PLoS One 12, e0179492 (2017).

4) Subramani, C.; Rajakkannu, A.; Rathinam, A.; Gaidhani, S.; Raju, I.; Kartar Singh, D.V. Anti-atherosclerotic activity of root bark of Premna integrifolia Linn. in high fat diet induced atherosclerosis model rats. $J$. Pharm. Anal. 7, 123-128(2017).

5) Wan, J.; Feng, Y.; Du, L.; Veeraraghavan, V.P.; Mohan, S.K.; Guo, S. Antiatherosclerotic activity of eriocitrin in high-fat-diet-induced atherosclerosis model rats. $J$. Environ. Pathol. Toxicol. Oncol. 39, 61-75(2020).

6) Lu, J.X.; Guo, C.; Ou, W.S.; Jing, Y.; Niu, H.F.; Song, P.; Li, Q.Z. et al. Citronellal prevents endothelial dysfunction and atherosclerosis in rats. J. Cell Biochem.120, 3790-3800 (2019).

7) Cao, H.; Jia, Q.; Shen, D.; Yan, L.; Chen, C.; Xing, X. Quercetin has a protective effect on atherosclerosis via enhancement of autophagy in ApoE - / - mice. Exp. Ther. Med. 18, 2451-2458(2019).

8) Wu, J.; Zhang, H.; Zheng, H.; Jiang, Y. Hepatic inflammation scores correlate with common carotid intimamedia thickness in rats with NAFLD induced by a high-fat diet. BMC Vet. Res. 10, 162 (2014).

9) Pang, J.; Xu, Q.; Xu, X.; Yin, H.; Xu, R.; Guo, S.; Hao, W. et al. Hexarelin suppresses high lipid diet and vitamin D3-induced atherosclerosis in the rat. Peptides 31, 630-638 (2010).

10) Ma, Q.; Yang, Q.; Chen, J.; Yu, C.; Zhang, L.; Zhou, W.; Chen, M. Salvianolic acid A ameliorates early-stage atherosclerosis development by inhibiting NLRP3 inflammasome activation in Zucker diabetic fatty rats. Molecules 25, 1089 (2020).

11) Masdar, H.; Hamidy, M.Y.; Darmawi; Trihardi, R.; Perwira, A.; Utari, D. Anti-atherosclerotic effects of Sonneratia Alba fruit extract in atherosclerotic-induced rats. Int. J. Appl. Pharm. 12, 41-43(2020).
12) Lian, N.; Tong, J.; Li, W.; Wu, J.; Li, Y. Ginkgetin ameliorates experimental atherosclerosis in rats. Biomed. Pharmacother. 102, 510-516 (2018).

13) Luo, Y.; Fang, J.L.; Yuan, K.; Jin, S.H.; Guo, Y. Ameliorative effect of purified anthocyanin from Lycium ruthenicum on atherosclerosis in rats through synergistic modulation of the gut microbiota and NF- $\mathrm{BB} /$ SREBP-2 pathways. J. Funct. Foods 59, 223-233 (2019).

14) Zhang, Y.; Liu, Z.; Zhou, M.; Liu, C. Therapeutic effects of fibroblast growth factor-21 against atherosclerosis via the NF-кB pathway. Mol. Med. Rep. 17, 1453-1460 (2018).

15) Conti, L.C.; Segura-Egea, J.J.; Cardoso, C.B.M.; Benetti, F.; Azuma, M.M.; Oliveira, P.H.C.; Bomfim, S.R.M. et $a l$. Relationship between apical periodontitis and atherosclerosis in rats: lipid profile and histological study. Int. Endod. J. 53, 1387-1397(2020).

16) Diao, S.L.; Sun, J.W.; Ma, B.X.; Li, X.M.; Wang, D. Influence of crocetin on high-cholesterol diet induced atherosclerosis in rats via anti-oxidant activity together with inhibition of inflammatory response and p38 MAPK signaling pathway. Saudi. J. Biol. Sci. 25, 493499 (2018)

17) Yang, Y.B.; Li, P.; Liu, M.L. Effects of n-3 polyunsaturated fatty acids on hemorheology and coagulation in atherosclerotic rats. Natl. Med. J. China 90, 20042007 (2010).

18) Hu, Y.; Sun, B.; Liu, K.; Yan, M.; Zhang, Y.; Miao, C.; Ren, L. Icariin attenuates high-cholesterol diet induced atherosclerosis in rats by inhibition of inflammatory response and p38 MAPK signaling pathway. Inflammation 39, 228-236 (2016).

19) Liu, C.; Sun, E.; Meng, W.; Sun, G. Protective effect of crocetin from crocus sativus l. On myocardial ischemia-reperfusion injury in rats. Food Sci. Technol. 39, 595-600 (2019).

20) Zhang, C.; Chen, K.; Wang, J.; Zheng, Z.; Luo, Y.; Zhou, W.; Zhuo, Z. et al. Protective effects of crocetin against radiation-induced injury in intestinal epithelial cells. Biomed. Res. Int. 2020, 2906053 (2020).

21) Ohba, T.; Ishisaka, M.; Tsujii, S.; Tsuruma, K.; Shimazawa, M.; Kubo, K.; Umigai, N. et al. Crocetin protects ultraviolet A-induced oxidative stress and cell death in skin in vitro and in vivo. Eur. J. Pharmacol. 789, 244-253 (2016).

22) Liu, Y.; Liang, Y.; Zheng, B.; Chu, L.; Ma, D.; Wang, H.; Chu, X. et al. Protective effects of crocetin on arsenic trioxide-induced hepatic injury: Involvement of suppression in oxidative stress and inflammation through activation of nrf2 signaling pathway in rats. Drug Des. Devel. Ther. 14, 1921-1931 (2020).

23) Zhou, C.; Bai, W.; Chen, Q.; Xu, Z.; Zhu, X.; Wen, A.; Yang, X. Protective effect of crocetin against burn-in- 
duced intestinal injury. J. Surg. Res. 198, 99-107 (2015).

24) Li, Y.; Kakkar, R.; Wang, J. In vivo and in vitro approach to anti-arthritic and anti-inflammatory effect of crocetin by alteration of nuclear factor-E2-related factor 2/hem oxygenase (HO) -1 and NF-кB expression. Front. Pharmacol. 9, 1341 (2018).

25) Saravanan, S.; Srikumar, R.; Manikandan, S.; Jeya Parthasarathy, N.; Sheela Devi, R. Hypolipidemic effect of triphala in experimentally induced hypercholesteremic rats. Yakugaku Zasshi 127, 385-388 (2007).

26) Jegadeesh, R.; Raaman, N.; Hariprasath, L.; Ramesh, V.; Srikumar, R. Hypolipidemic effect of Pleurotus djamor var. roseus in experimentally induced hypercholesteromic rats. Res. J. Pharm. Biol. Chem. Sci. 5, 581-588(2014).

27) Li, Q.; Wang, H.; Zhang, C.; Tong, R.; Chen, H.; Qie, R. Ethyl acetate extract of sappanwood alleviates experimental atherosclerosis in rats through changes in FGF21 and SREBP-2 expression. Int. J. Clin. Exp. Pathol. 13, 220-229 (2020).

28) Hong-zhen, Z.; Jiao, R.; Li, L.; Zhang, Y.; Qian, Y.; Chunlan, G. Effects of Qi-benefiting blood-activating Chinese herbs on aorta Rho kinase and related cytokine expression in a rat model of atherosclerosis. Chinese J. Tissue Eng. Res. 20, 2703-2710 (2016).

29) Sethwala, A.M.; Goh, I.; Amerena, J.V. Combating inflammation in cardiovascular disease. Hear. Lung Circ. 30, 197-206 (2021).

30) Harb, A.A.; Bustanji, Y.K.; Abdalla, S.S. Hypocholesterolemic effect of $\beta$-caryophyllene in rats fed cholesterol and fat enriched diet. J. Clin. Biochem. Nutr. Published online 2018. doi:10.3164/jcbn.17-3

31）Thatiparthi, J.; Dodoala, S.; Koganti, B.; Kvsrg, P. Barley grass juice (Hordeum vulgare L.) inhibits obesity and improves lipid profile in high fat diet-induced rat model. J. Ethnopharmacol. 238, 111843(2019).

32) Lin, C.H.; Xu, H.L.; Sun, W.L.; Li, F.; Lin, G.Z. Equisetum sylvaticum base reduces atherosclerosis risk factors in rats fed a high-fat diet. Bangladesh J. Phar- macol. 9, 257-261 (2014).

33) He, K.; Wang, J.; Shi, H.; Yu, Q.; Zhang, X.; Guo, M.; Sun, H. et al. An interspecies study of lipid profiles and atherosclerosis in familial hypercholesterolemia animal models with low-density lipoprotein receptor deficiency. Am. J. Transl. Res. 11, 3116-3127 (2019).

34) Othman, Z.A.; Zakaria, Z.; Suleiman, J.B.; Ghazali, W.S.W.; Mohamed, M. Anti-atherogenic effects of orlistat on obesity-induced vascular oxidative stress rat model. Antioxidants 10, 251 (2021).

35) Endo, A. The discovery and development of HMG-CoA reductase inhibitors. J. Lipid Res. 33, 1569-1582 (1992).

36) Oliveira, E.F.; Santos-Martins, D.; Ribeiro, A.M.; Brás, N.F.; Cerqueira, N.S.; Sousa, S.F.; Ramos, M.J. et al. HMG-CoA Reductase inhibitors: An updated review of patents of novel compounds and formulations (20112015). Expert Opin. Ther. Pat. 26, 1257-1272 (2016).

37) Xu, X.; Ma, Q.; Qin, H.; Zhang, J.; Zhang, B.; Pang, H.; Yin, Q. et al. Anhuienoside $\mathrm{C}$ ameliorates atherosclerosis in rats via regulation of the $\mathrm{NF \kappa B} / \mathrm{eNOS} / \mathrm{NO}$ signaling pathway. Trop. J. Pharm. Res. 19, 721-726 (2020).

38) Nakashima, Y.; Raines, E.W.; Plump, A.S.; Breslow, J.L.; Ross, R. Upregulation of VCAM-1 and ICAM-1 at atherosclerosis-prone sites on the endothelium in the apoE-deficient mouse. Arterioscler. Thromb. Vasc. Biol. 18, 842-851 (1998).

39) Förstermann, U.; Sessa, W.C. Nitric oxide synthases: Regulation and function. Eur. Heart J. 33, 829-837 (2012).

CC BY-SA 4.0 (Attribution-ShareAlike 4.0 International). This license allows users to share and adapt an article, even commercially, as long as appropriate credit is given and the distribution of derivative works is under the same license as the original. That is, this license lets others copy, distribute, modify and reproduce the Article, provided the original source and Authors are credited under the same license as the original. 